\title{
Prevalence of Extended Spectrum $\beta$-Lactamases (ESBL) Producers Among Gram-Negative Bacilli in Wound Infection
}

\author{
Abu Hena Md. Saiful Karim Chowdhury ${ }^{1 *}$ \\ Md Anwar Husain ${ }^{2}$ \\ Nasima Akter ${ }^{2}$ \\ Khan Mashrequl Alam ${ }^{2}$ \\ Arup Kanti Dewanjee ${ }^{2}$ \\ Shakeel Ahmed ${ }^{2}$ \\ Zakir Hossain ${ }^{2}$ \\ Arifur Rahman ${ }^{2}$ \\ Towhida Naheen ${ }^{3}$ \\ Md Abdul Mazed ${ }^{4}$ \\ Sanjoy Kanti Biswas ${ }^{5}$ \\ Md Ehsanul Hoque ${ }^{6}$ \\ 'Department of Pathology \\ Chittagong Medical College \\ Chittagong, Bangladesh. \\ ${ }^{2}$ Department of Microbiology \\ Chittagong Medical College \\ Chittagong, Bangladesh. \\ ${ }^{3}$ Department of Anatomy \\ University of Science \& \\ Technology Chittagong (USTC) \\ Chittagong, Bangladesh. \\ ${ }^{4}$ Department of Microbiology \\ Cox's Bazar Medical College \\ Cox's Bazar, Bangladesh. \\ ${ }^{5}$ Department of Microbiology \\ Chattagram Maa-O-Shisu Hospital \\ Medical College \\ Chittagong, Bangladesh. \\ ${ }^{6}$ Department of Microbiology \\ Rangamati Medical College \\ Rangamati, Bangladesh. \\ *Correspondence to:
}

Dr. Abu Hena Md Saiful Karim Chowdhury

Clinical Pathologist

Department of Pathology

Chittagong Medical College

Chittagong, Bangladesh.

Mobile: +8801819636883

E-mail:drsaifulkarim@yah.com

www.banglaiol.info/index.php/CMOSHMCJ

\begin{abstract}
Background: Extended Spectrum $\beta$-Lactameses Producing Organisms (ESBLs) are increasing in number and causing more severe infections because of their continuous mutation and multidrug resistance property with limited therapeutic option. Aims and Objectives: Present study was undertaken to detect the prevalence of the ESBLs producing bacteria in wound infection, so as to provide a base line data in treating them \& prevent unnecessary use of antibiotics Methods: Isolated gram-negative bacteria initially screened by Minimum Inhibitory Concentration (MIC) ESBLs breakpoints. Then suspected ESBLs producers were confirmed by Phenotypic confirmatory test. Results: 105 (One hundred five) (91.30\%) bacterial strains were isolated from 115 samples of wound swab \& pus from different patients were studied of which $84(80.00 \%)$ were Gram-negative and 21(20.00\%) were Gram-positive. Among the isolated Gram-negative bacteria 79(94.05\%) were found suspected ESBLs producers of which 54(68.35\%) were found as confirmed ESBL producers. The prevalence of ESBLs producing organisms in the present study were found to be $64.29 \%$ and Klebsiella spp as most prevalent ESBLs producers. Conclusion: It is essential to report ESBL production along with routine sensitivity reporting, which will help the clinician in prescribing the proper antibiotics.
\end{abstract}

Key words : Gram-negative bacilli; ESBL; Klebsiella Species; Infection.

\section{INTRODUCTION}

Antimicrobial resistance is emerging as one of the major public health threats at the beginning of the $21^{\text {st }}$ century worldwide including Bangladesh. The wide spread use and in some cases, misuse of antimicrobials in all health care settings over the past several decades has been cited as a contributing factor in the development of drug resistance in virtually all bacterial species. The accelerated emergence of antibiotic resistance among the prevalent pathogens is the most serious threat to the management of infectious diseases ${ }^{1}$. Infections by extended spectrum beta lactamases (ESBLs) producing organisms are causing significant diagnostic and therapeutic problems in affected patient's ${ }^{2}$. ESBLs are mutant forms of $\beta$-lactamases enzymes coded by genes located on transferable plasmids, which can easily spread form one organism to another. The ESBL producing organisms are often multi drug resistant, as the plasmids producing ESBLs can carry resistance to other antibiotics ${ }^{3}$. Extended spectrum $\beta$-lactamases producing bacteria produce Extended Spectrum $\beta$-Lactamases (ESBLs) enzymes that mediate resistance to extended spectrum (Third generation) cephalosporins and monobactams but do not affect cephamycins (e.g. Cefoxitin and cefotatan) or carbapenems and are inhibited by $\beta$-lactamase inhibitors such as clavulanate, sulbactam and tazobactam ${ }^{1,4,5}$. 
The ESBL producing bacteria are increasingly becoming a major threat for patients in the hospital, long term care facilities and community. The increasing drug resistance among these bacteria has made therapy difficult and has led to a greater use of expensive broad spectrum antibiotics ${ }^{6}$. Inappropriate antibiotic selection in infections caused by these organisms is associated with treatment failures, poor clinical outcomes, prolonged hospital stay, increased morbidity, mortality and health care costs. The widespread use of the third generation cephalosporins and aztreonam is believed to be the major cause of the mutations in these enzymes ${ }^{7}$. Drug resistance of this form is often difficult to recognize using conventional antimicrobial susceptibility methods. Failure to identify ESBL producing organisms also contributes to their uncontrolled spread. Therefore, identification of the resistant phenotypes is important, particularly in developing countries where there is excessive use of antibiotics and lack of adequate antimicrobial resistance surveillance ${ }^{8,9}$.

This study was designed to investigate the prevalence of ESBLs producing organisms among wound infections which would guide clinicians and microbiologists for proper handling of these pathogens \& prevent unnecessary use of antibiotics.

\section{MATERIALS AND METHODS}

This cross sectional study was carried out in the Department of Microbiology, Chittagong Medical College, during the period of June 2008 to May 2009. Samples were collected after taking informed written consent from patients admitted in different wards of Chittagong Medical College Hospital, Chittagong.

Inclusion criteria: The following categories of patients were included in this study:

i. Patients with infected wound.

ii. Infected burn patients.

Exclusion criteria: Newly admitted burn/surgical cases.

A sterile technique was applied to aspirate or collect pus or wound swab from abscess or wound infection, either by disposable syringe or by sterile swab stick and inoculated in Blood agar and Mac Conkey agar media. After overnight incubation at $37^{\circ} \mathrm{C}$, plates were checked for presence of any suspected pathogens. Identification of organisms were done as per standard laboratory methods of identification and antimicrobial sensitivity of the isolates were tested against different antibiotics.

ESBL detection: The method recommended by Clinical Laboratories Standard Institute (CLSI) requires a two step approach of initially screening for ESBL production and then performing confirmatory tests on screen positive isolates ${ }^{10}$.

Screening for ESBL producers by dilution method

Agar dilution method: The screening for ESBL producers was done by agar dilution method as was recommended by CLSI. Any of the isolated organisms found to be grown at this stated screening antibiotics concentration (That is Minimum
Inhibitory Concentration [(MIC)] of third generation cephalosporins, namely ceftriaxone, ceftazidime and cefotaxime $>2 \mu \mathrm{g} / \mathrm{ml}$ ) according to CLSI, 2007 was considered as possible ESBL producers and spelled for the confirmatory tests. The use of more than one antimicrobial agent for screening improves the sensitivity of detection ${ }^{10}$.

Detection of ESBLs by the confirmatory tests

Phenotypic confirmatory test: Confirmation of the ESBL producing isolates was done by the phenotypic confirmatory test according to CLSI recommendation. In this test, third generation cephalosporin i.e. ceftazidime $(30 \mu \mathrm{g})$ and cefotaxime $(30 \mu \mathrm{g})$ disc alone and in combination with clavulanic acid $(10 \mu \mathrm{g})$ were used. Ceftazidime, cefotaxime discs were placed on one side and ceftazidime, cefotaxime discs combined with clavulanic acid $(30 / 10 \mu \mathrm{g})$ were placed on other side of the inoculated plate. After overnight incubation at $37 \mathrm{C}$, diameter of zone of inhibition was measured. A $5 \mathrm{~mm}$ or more increases in diameter of zone of inhibition for ceftazidime and cefotaxime tested in combination with clavulanic acid versus its zone when ceftazidime and cefotaxime tested alone confirms an ESBLs producing organism ${ }^{10}$.

Reference strain for quality control used for ESBL detection

E. coli BB-32327 (CTX-M9) was used as positive control and E. coli ATCC (American Type Culture Collection) 25922 was used as negative control of ESBL detection test.

\section{RESULTS}

A total 115 samples of wound swab \& pus from different patients were studied, of which $105(91.30 \%)$ bacterial strains were isolated \& $10(08.70 \%)$ found no growth (Figure 1).

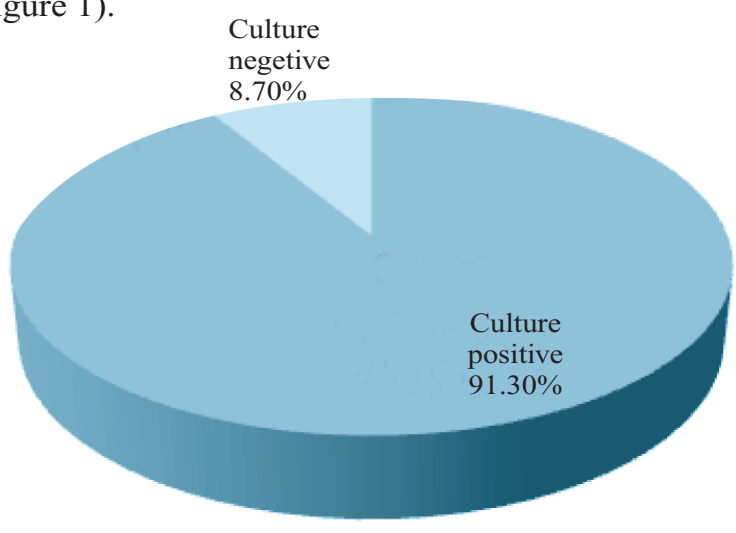

Figure 1: Frequencies of isolates from wound infection.

Among the 105 bacterial isolates 84(80.00\%) were Gramnegative and $21(20.00 \%)$ were Gram-positive, of which majority were Klebsiella spp 26(24.76\%) followed by E. Coli 25(23.81\%) Pseudomonas spp 23(21.90\%) Staphylococcus aureus 18(17.14) Proteus spp 10(09.53\%) $\&$ Coagulase negative Staphylococci 03(2.86). (Table 1). 
Table 1 : Distribution of bacterial species among the isolates $(\mathrm{n}=105)$.

$\begin{array}{lcc}\text { Name of bacterial species } & \text { Number(n=105) } & \text { Percentage } \\ \text { Gram-negative organism } & & \\ \text { Klebsiella } \text { species } & 26 & 24.76 \\ \text { E. Coli } & 25 & 23.81 \\ \text { Pseudomonas species } & 23 & 21.90 \\ \text { Proteus species } & 10 & 09.53 \\ \text { Total } & 84 & 80.00 \\ \text { Gram-positive organism } & & \\ \text { Staphylococcus aureus } & 18 & 17.14 \\ \text { Coagulase negative } & & \\ \text { Staphylococci } & 03 & 02.86 \\ \text { Total } & 21 & 20.00 \\ \text { Grand Total } & \mathbf{1 0 5} & \mathbf{1 0 0 . 0 0}\end{array}$

It appears from Figure 2 that total 84 isolated gram-negative bacteria were screened for suspected ESBLs producers on the basis of MIC ESBL breakpoints by agar dilution method, out of which $79(94.05 \%)$ were found suspected ESBLs producers \& 05(05.95\%) gave negative result.

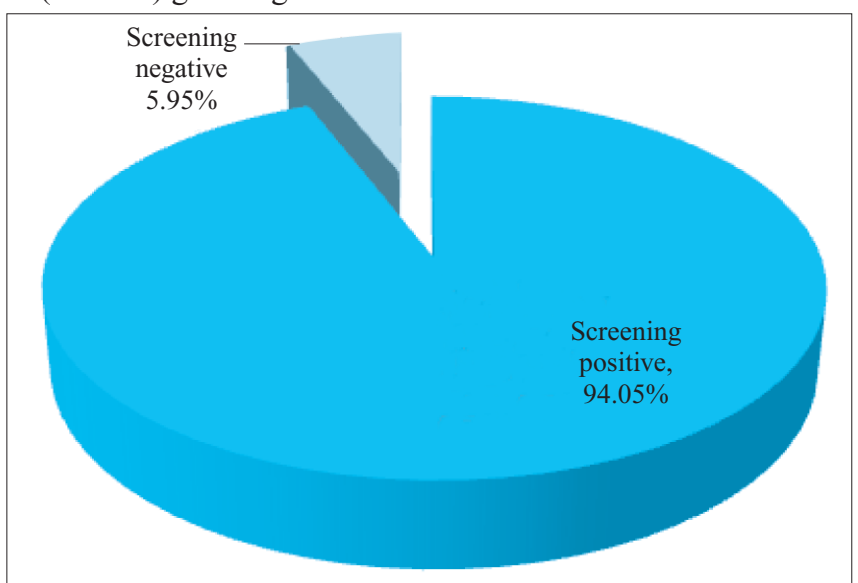

Figure 2 : Detection of ESBL producing bacteria on the basis of MIC (Screening test) by agar dilution method.

Screening positive 79 suspected ESBLs producing bacteria were subjected to phenotypic confirmatory test, $54(68.35 \%)$ were found as confirmed ESBL producers \& 25(31.65\%) showed negative result (Figure 3).

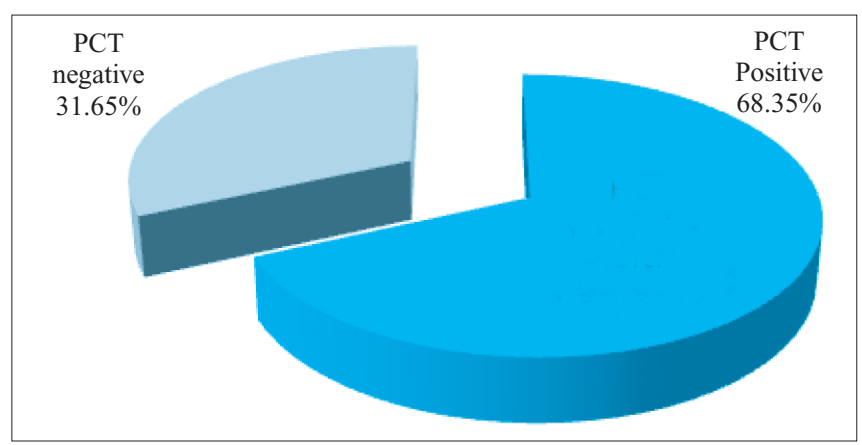

Figure 3: Detection of ESBL producing organisms on the basis of phenotypic confirmatory test.
Out of 84 gram-negative bacteria 54(64.29\%) were found to ESBLs producer. Higher rate of ESBLs was observed in Klebsiella spp $19(73.03 \%)$ out of 26, followed by E. Coli $17(68.00 \%)$ out of 25 , Pseudomonas spp $13(56.52 \%)$ out of 23 \& Proteus spp 05(50.00\%) out of 10 (Table 2).

Table 2 : Distribution of ESBLs producers among gram-negative bacteria $(n=84)$.

\begin{tabular}{lcc}
$\begin{array}{l}\text { Name of } \\
\text { bacteria }\end{array}$ & $\begin{array}{c}\text { Total no. of gram- } \\
\text { negative bacteria }\end{array}$ & $\begin{array}{c}\text { Number of ESBL } \\
\text { producers }\end{array}$ \\
Klebsiella species & 26 & $19(73.03)$ \\
E. Coli & 25 & $17(68.00)$ \\
Pseudomonas species & 23 & $13(56.52)$ \\
Proteus species & 10 & $05(50.00)$ \\
Total & 84 & $54(64.29)$ \\
\hline
\end{tabular}

- Figures within parentheses indicate percentages

\section{DISCUSSION}

In the present study, 105(91.30\%) bacteria were isolated from 115 wound swab \& pus samples from different patients (Figure 1). This result is closely related to that of Rahman in Bangabandhu Sheikh Mujib Medical University(BSMMU) Dhaka, who found $93.92 \%$ bacterial isolates from wound swab $\&$ pus samples ${ }^{11}$.

Among the bacterial isolates, 84(80.00\%) were gram-negative and $21(20.00 \%)$ were gram-positive in our study (Table 1). Similar to present study, Rahman found $90 \%$ gram-negative \& $10 \%$ gram-positive isolates ${ }^{11}$. Amongst the isolates, Klebsiella spp $(24.76 \%)$ was the predominant organism, followed by $E$. Coli $(23.81 \%)$ Pseudomonas spp (21.90\%) Staphylococcus aureus (17.14\%) Proteus spp (9.53\%) and Coagulase-negative staphylococci $(2.86 \%)$. Sule et al. in Nigeria also found Klebsiella spp (25.3\%) as the most common bacterial isolates from wound swab ${ }^{12}$. In contrast to present study Rahman et al. of Dhaka, found Staphylococcus aureus (39.44\%) was the principal organism, followed by Klebsiella spp (21.13\%) E. Coli $(11.27 \%)$ Proteus spp (08.45\%) and Pseudomonas spp $(05.63 \%)$ in surgical wound ${ }^{13}$. These different findings may be due to that etiological agents may however, vary from country to country, from hospital to hospital and from one community to another ${ }^{14}$.

As of now, no country wide study has been conducted for the detection of the prevalence of ESBL production in Bangladesh, individual studies which were done in different parts of the country showed a varying prevalence, based on various risk factors and local reasons.

In the present study, we found $79(94.05 \%)$ suspected ESBLs producers from 84 gram-negative isolates, based on Minimum Inhibitory Concentration (MIC) ESBLs screening breakpoints (Figure 2). As using more than one antibiotic increase the sensitivity, we used three third generation cephalosporins (Ceftriaxone, ceftazidime \& cefotaxime) for the screening ${ }^{10}$. 
Our finding is closely related to that of Metri et al. in North Karnataka, India, who found $91.74 \%$ suspected ESBLs producers by screening test ${ }^{7}$. When these 79 screening positive isolates were subjected to the confirmatory tests, 54(68.35\%) were confirmed as ESBL producers by phenotypic confirmatory test (Figure 3 ).

The prevalence of ESBLs producing organisms in the present study were found (Table II) to be $64.29 \%$, which is higher than that of Alim 23.19\% and Rahman 30.90\% both in BSMMU but lower than that of Biswas of BSMMU $80 \%$ and Yasmin of Mymensingh $71.30 \%{ }^{15}, 11,16,17$. Ullah et al. in Pakistan found $58.7 \%$ ESBL producers ${ }^{18}$. The prevalence of ESBLs producers in India ranges from $6.6 \%$ to $91 \%$, in Europe from $23-25 \%$ for Klebsiella spp and 5.4\% for E. Coli and in United States from 0 to $25 \%$, depending on the institution ${ }^{7,3}$.

The variation on ESBL positivity might be due to the number of isolates studied, variation in institution to institution \& geographic location ${ }^{5,8}$. The prevalence of ESBL production is high in the referral centers and the intensive care unites where the patients are referred from the peripheral centers and where the antibiotic use is profuse ${ }^{7}$. The uncontrolled use of 3rd generation cephalosporins at the hospital could be a leading contributory factor to the high ESBLs prevalence ${ }^{19}$.
ESBLs are most commonly recognized in Klebsiella spp and E. Coli and most prevalent in Klebsiella pneumoniae ${ }^{20,21}$. We also found Klebsiella spp (73.03\%) as the leading ESBLs producers followed by E. Coli (68.00\%) Pseudomonas spp (50.00\%) and Proteus spp (50.00\%) in our study, which correlates with those of Rahman \& Alim both in BSMMU, Yasmin of Mymensingh who also found Klebsiella spp as the most common ESBL producers $^{11,15,17}$.

The high occurrence of ESBLs in Klebsiella spp is of great concern since infections caused by this bacterium are very common and resistance of the organism may be due to the presence of capsule \& multidrug resistance efflux pump, easy spreading nature, pathogenic and efficient at acquiring and disseminating resistance plasmid and production of endotoxin, carbapenemases, which make it more resistant $\mathrm{t}^{22}$.

\section{CONCLUSION}

Existing of extended spectrum $\beta$-lactameses in bacteria and their potential multidrug resistance will create serious problem in the future as their continuous mutation and limited therapeutic option. Indiscriminate use of antibiotics especially 3rd generation cephalosporins and monobactams should be avoided. The regular detection of ESBLs producing organisms should be carried out in every laboratory.

\section{DISCLOSURE}

All the authors declared no competing interest. 


\section{REFERENCES}

1. $\quad$ Al-Jasser AM. Extended-Spectrum $\beta$-Lactamases (ESBLs): A global problem. Kuwait Med J. 2006; 38: 171-185.

2. Livermore DM. $\beta$-lactamases in laboratory and clinical resistance. Clinical Microbiology Reviews. 1995; 8(4): 557-584.

3. Sirot D. Extended-spectrum plasmid mediated beta-lactamases. J Antimicrob Chemother. 1995; 36 (Suppl A): 19-34.

4. $\quad$ Paterson DL, Bonomo RA. Extended-spectrum B-lactamases: A clinical update, Clinical Microbiology Reviews. 2005; 18(4):657-686.

5. Bradford PA. Extended Spectrum $\beta$-Lactamases in the 21st century: Characterization, epidemiology, and detection of this important resistant threat. Clinical Microbiology Reviews. 2001; 14(4): 933-951.

6. Kader AA, Angamuthu K, Islam KMS. Extended Spectrum $\beta$-Lactamases in urinary isolates of Escherichia coli, Klebsiella pneumoniae and other gram-negative bacteria in a hospital in Eastern Province, Saudi Arabia. Saudi Med J. 2005; 26(6): 956-959.

7. Metri BC, Jyothi P, Peerapur BV. The prevalence of ESBL among Enterobaceriaceae in a tertiary care hospital of North Karnataka, India. $J$ of Clinical and Diagnostic Research. 2011; 5(3): 470-475.

8. Kader AA, Kumar A, Krishna A, Zaman MN. An Accelerated method for the detection of Extended Spectrum $\beta$-Lactamases in urinary isolates of Escherichia coli, Klebsiella pneumoniae. Saudi J Kidney Dis Transpl. 2006; 17(4): 535-539.

9. Sobel JD, Kaye D. Urinary Tract Infections in GL Mandell, JE Bennett \& R Dolin (eds), Mandell, Doglus and Bennett's principles and practice of infectious diseases, Churchill Livingstone, Philadelphia. 2010; 7:957-985.

10. Clinical and Laboratory Standards Institute. Performance standards for antimicrobial susceptibility testing, seventeenth informational supplement. CLSI Document M100-S17, Wayne, Pennsylvania, USA. 2007; 27(1): 1-177.

11. Rahman M. Rapid detection of extended-spectrum $\beta$-lactamases production directly from primary culture. M. Phil. Thesis, Bangabandhu Sheikh Mujib Medical University, Dhaka. 2007.

12. Sule AM, Thanni LOA, Sule-Odu OA, Olusanya O. Bacterial pathogens associated with infected wounds in Ogun State University Teaching Hospital, Saganu, Nigeria. African J of Clinical \& Experimental Microbiology. 2001; 3(1): 13-16.

13. Rahman MM, Haq JA, Hossain MA, Sultana R, Islam F, Islam AHMS. Prevalence of Extended Spectrum $\beta$-Lactamase producing Escherichia coli and Klebliella pneumoniae in an urban hospital in Dhaka, Bangladesh. International J of Antimicrobial Agents. 2004; 24(5): 508-510.

14. Kuruvilla AC. Neonatal septicemia. Indian J Pediatr. 1988; 55: 225-233.

15. Alim R. Detection of Extended Spectrum $\beta$-Lactamases (ESBLs) producing bacteria. M. Phil. Thesis, Bangabandhu Sheikh Mujib Medical University, Dhaka. 2005.

16. Biswas SM. Comparison of three dimensional test and double disc synergy test for detection of Extended Spectrum $\beta$-Lactamase (ESBL) producing gram-negative bacteria. M.Phil.Thesis, Bangabandhu Sheikh Mujib Medical University, Dhaka. 2009.

17. Yasmin T. Prevalence of ESBL among Esch. coli and Klebsiella spp in a tertiary care hospital and molecular detection of important ESBL producing genes by multiplex PCR. M.Phil. Thesis, Mymensingh Medical College, Mymensingh. 2012.

18. Ullah F, Malik.SA, Ahamed J. Antibiotic susceptibility pattern of ESBL prevalence in nosocomial Escherichia coli from urinary tract infections in Pakistan. African J of Biotechnology. 2009; 8(16): 3921-3926.

19. Ahamed K, Thokar MA, Toboli AS, Fomda BA, Bashir G, Maroof P. Extended Spectrum $\beta$-Lactamase mediated resistance in Escherichia coli in a tertiary care hospital in Kashmir, India. African J of Microbiology Rasearch. 2010; 4(24):2720-2728.

20. Bush K, Jacoby GA, Medeiros AA. A functional classification scheme for $\beta$ - Lactamases and its correlation with molecular structure. Antimicrobial Agents and Chemotherapy. 1995; 39(6): 1211-1233.

21. Tribuddharat C, Srifuengfung S, Chiangjong W. A correlation between phenotypes and genotypes of Extended Spectrum Beta-Lactamase (ESBL) producing Klebsiella pneumoniae in a university hospital, Thailand. J Infect Dis Antimicrob Agents. 2007; 24(3): 117-123.

22. Lin Y, Lu M, Tang H, Liu H, Chen C, Liu K et al. Assessment of hypermuco viscosity as a virulence factor for experimental Klebsiella pneumoniae infections: Comparative virulence analysis with hypermuco viscosity negative strain. BMC Microbiology. 2011; 11(50) viewed on 15 April 2012, http://www.biomedcentral.com/1471-2180/11/50. 